\title{
The CCAAT-binding complex mediates azole susceptibility of Aspergillus fumigatus by suppressing SrbA expression and cleavage
}

\author{
Chi Zhang ${ }^{1}$, Lu Gao ${ }^{1}$, Yiran Ren ${ }^{1}$, Huiyu Gu${ }^{1}$, Yuanwei Zhang ${ }^{1}$, and Ling Lu \\ ${ }^{1}$ Nanjing Normal University College of Life Sciences
}

November 1, 2021

\begin{abstract}
In fungal pathogens, the transcription factor SrbA (a sterol regulatory element-binding protein, SREBP) and CBC (CCAAT binding complex) have been reported to regulate azole resistance by competitively binding the TR34 region (34 mer) in the promoter of the drug target gene, erg11A. However, current knowledge about how the SrbA and CBC coordinately mediate erg11A expression remains limited. In this study, we uncovered a novel relationship between HapB (a subunit of CBC) and SrbA in which deletion of hapB significantly prolongs the nuclear retention of SrbA by increasing its expression and cleavage under azole treatment conditions, thereby enhancing Erg11A expression for drug resistance. Furthermore, we verified that loss of HapB significantly induces the expression of the rhomboid protease RbdB, Dsc ubiquitin E3 ligase complex, and signal peptide peptidase SppA, which are required for the cleavage of SrbA, suggesting that HapB acts as a repressor for these genes which contribute to the activation of SrbA by proteolytic cleavage. Together, our study reveals that CBC functions not only to compete with SrbA for binding to erg11A promoter region but also to affect SrbA expression, cleavage, and translocation to nuclei for the function, which ultimately regulate Erg11A expression and azole resistance.
\end{abstract}

\section{Hosted file}

MB032021070378.pdf available at https://authorea.com/users/443995/articles/543848-the-ccaatbinding-complex-mediates-azole-susceptibility-of-aspergillus-fumigatus-by-suppressingsrba-expression-and-cleavage 